\title{
LA FRAGMENTACIÓN JURÍDICO-INSTITUCIONAL COMO OBSTÁCULO PARA APLICAR EL PARADIGMA AMBIENTAL
}

\section{LEGAL-INSTITUTIONAL FRAGMENTATION AS AN OBSTACLE FOR THE ENVIRONMENTAL PARADIGM APPLICATION}

\author{
Clara María Minaverry ${ }^{1}$ \\ cminaverry@derecho.uba.ar \\ Mariano Ferro ${ }^{2}$ \\ marianoferro@derecho.uba.ar
}

Fecha de recepción: 15 de marzo de 2016 - Fecha de aceptación: 27 de julio de 2016

\begin{abstract}
Resumen
Desde la incorporación de la cuestión ambiental en la agenda internacional, se generaron expectativas acerca de la disciplina del Derecho como herramienta para mitigar, prevenir y resarcir el daño antropogénico a los ecosistemas. En este artículo se abordará una cuestión poco tratada en la literatura de las ciencias jurídicas y sociales en temas ambientales: la fragmentación institucional y normativa como obstáculo para alcanzar mayor sostenibilidad, específicamente, en la gestión del agua. Se ha adoptado un diseño de tipo bibliográfico-documental, descriptivo y diacrónico. La metodología utilizada es cualitativa, en particular la hermenéutica jurídica. En forma preliminar, se concluye que el caos normativo aplicable a la Cuenca Matanza-Riachuelo ha dado lugar a arreglos institucionales fallidos generando un escenario adverso para la aplicación del paradigma ambiental en varias dimensiones: al favorecer la ocurrencia de acciones de corrupción; por dificultar el acceso a la información y el derecho a peticionar ante las autoridades; por constituir un obstáculo al ejercicio de la participación ciudadana.

Palabras clave: Cuenca Matanza-Riachuelo, Gestión fragmentada del agua, Participación ciudadana, Solapamiento normativo, Argentina.
\end{abstract}

\begin{abstract}
Since environmental topic was included in the international agenda, expectations were created about the role of Law as a discipline and tool to mitigate, prevent and compensate anthropogenic ecosystem damages. In this paper there will be an approach to an issue which was little addressed in social, legal and environmental sciences: legal-institutional fragmentation as an obstacle to achieve sustainable development goals, especially in water management. It was implemented as a documental-bibliographical, descriptive and diachronic design. The methodology used is qualitative,
\end{abstract}

1 Profesora Adjunta Regular de Derecho Ambiental (Universidad Nacional de Luján) y de posgrado en la Universidad Tecnológica Nacional y en la Universidad Católica Argentina. Investigadora del Consejo Nacional de investigaciones científicas y técnicas (CONICET) y del Instituto de investigaciones Jurídicas y Sociales Ambrosio Gioja, Facultad de Derecho, Universidad de Buenos Aires.

2 Profesor en la Facultad de Derecho de la Universidad de Buenos Aires, en el Instituto Superior de Enseñanza del Ejército (IESE) y en la Escuela de Guerra Naval (ESGN) y en la Universidad de Ciencias Empresariales y Sociales (UCES) en la Materia Recursos Estratégicos. Investigador del Instituto de Ciencias Jurídicas y Sociales Ambrosio Gioja, por la Facultad de Derecho, Universidad de Buenos Aires. 
mainly legal hermeneutics. Preliminary, we concluded that regulatory chaos which was applied to the Matanza-Riachuelo basin, allowed failed institutional settlements creating an adverse scenery for the environmental paradigm application in different dimensions: to favour corrupted actions, to difficult information access and authorities petitions right, because they both represent an obstacle to achieve citizen's participation.

Key Words: Matanza-Riachuelo Basin; Water fragmented management; Citizen's participation; Regulatory overlap; Argentina

\section{Introducción}

La Cuenca Matanza-Riachuelo (CMR) comparte un conjunto de características socio-ambientales y topográficas en común con el resto de las cuencas de la Región Metropolitana de Buenos Aires (RMBA): su topografía es relativamente plana y uniforme, típica de los ríos de llanura.

La CMR, junto con la Cuenca del Río Reconquista y la del Río Luján son las tres cuencas más importantes que atraviesan la RMBA. Sin embargo, por su densa imbricación con el desarrollo urbano durante el s. XX, se considera a la CMR como la de mayor relevancia regional (Malpartida, 2002, p. 4).

Mapa 1

Cuencas Metropolitanas de Buenos Aires

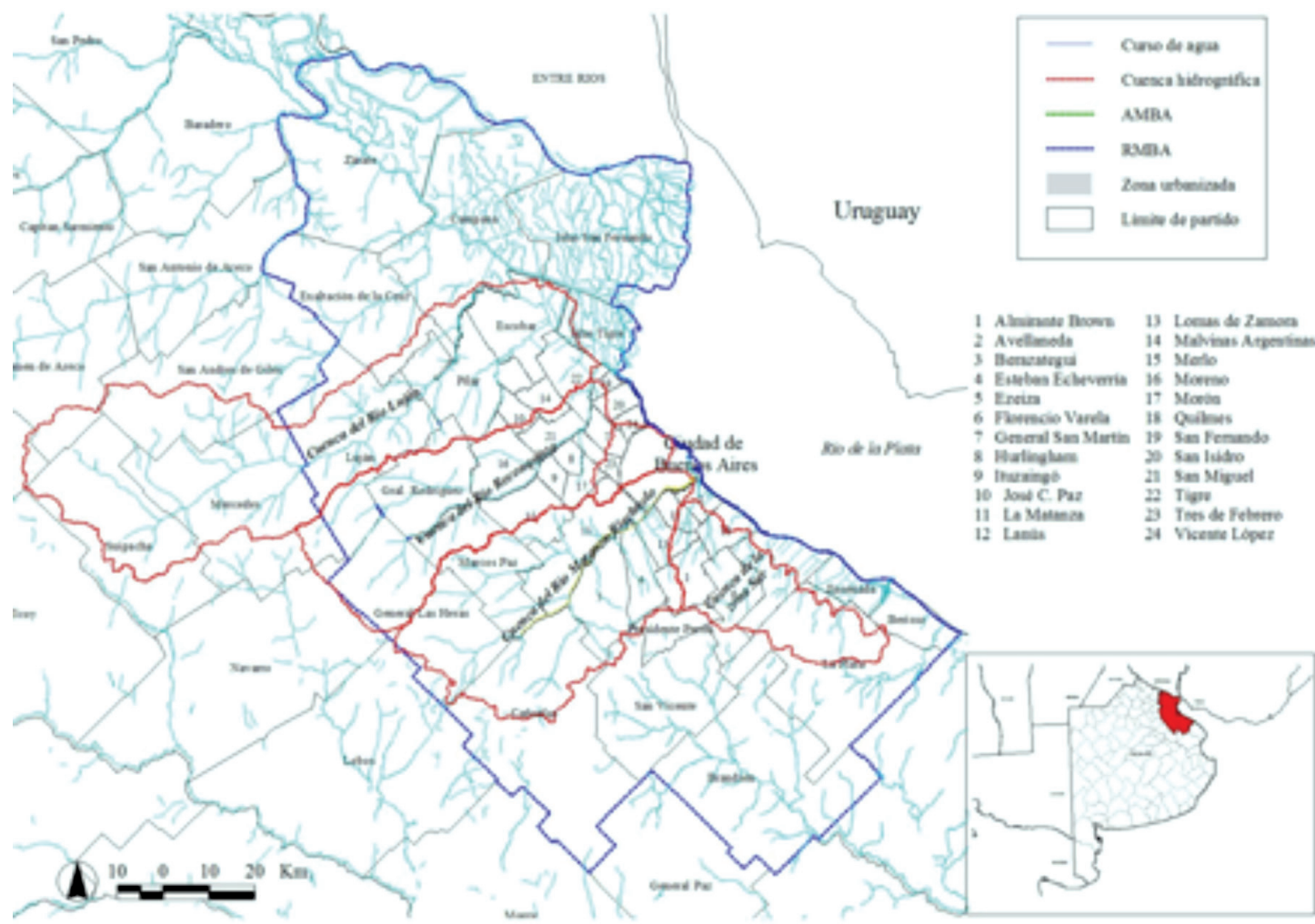

Fuente: Herrero \& Fernández (2008, p.19).

La CMR se ubica al este de Argentina y al noreste de la provincia de Buenos Aires, tiene una longitud aproximada de $70 \mathrm{~km}$ de extensión del sistema hídrico integrado por los ríos Matanza y Riachuelo, y cubre una superficie de $2200 \mathrm{Km}^{2}$ desde su nacimiento hasta la desembocadura en el Río de La Plata. 
Su nombre desde su naciente es Río Matanza. Desde el Puente de la Noria donde comienza la Av. Gral. Paz, que delimita a toda la Ciudad Autónoma de Buenos Aires, hasta su desembocadura su nombre es Riachuelo. Dentro de la CMR se pueden distinguir tres áreas: Cuenca Alta, Cuenca Media y Cuenca Baja.

Mapa 2

Ubicación geográfica de los 14 Partidos que conforman la Cuenca Matanza Riachuelo y la Ciudad Autónoma de Buenos Aires (CABA). Cuenca Alta (rojo), Media (naranja) y Baja (amarillo)

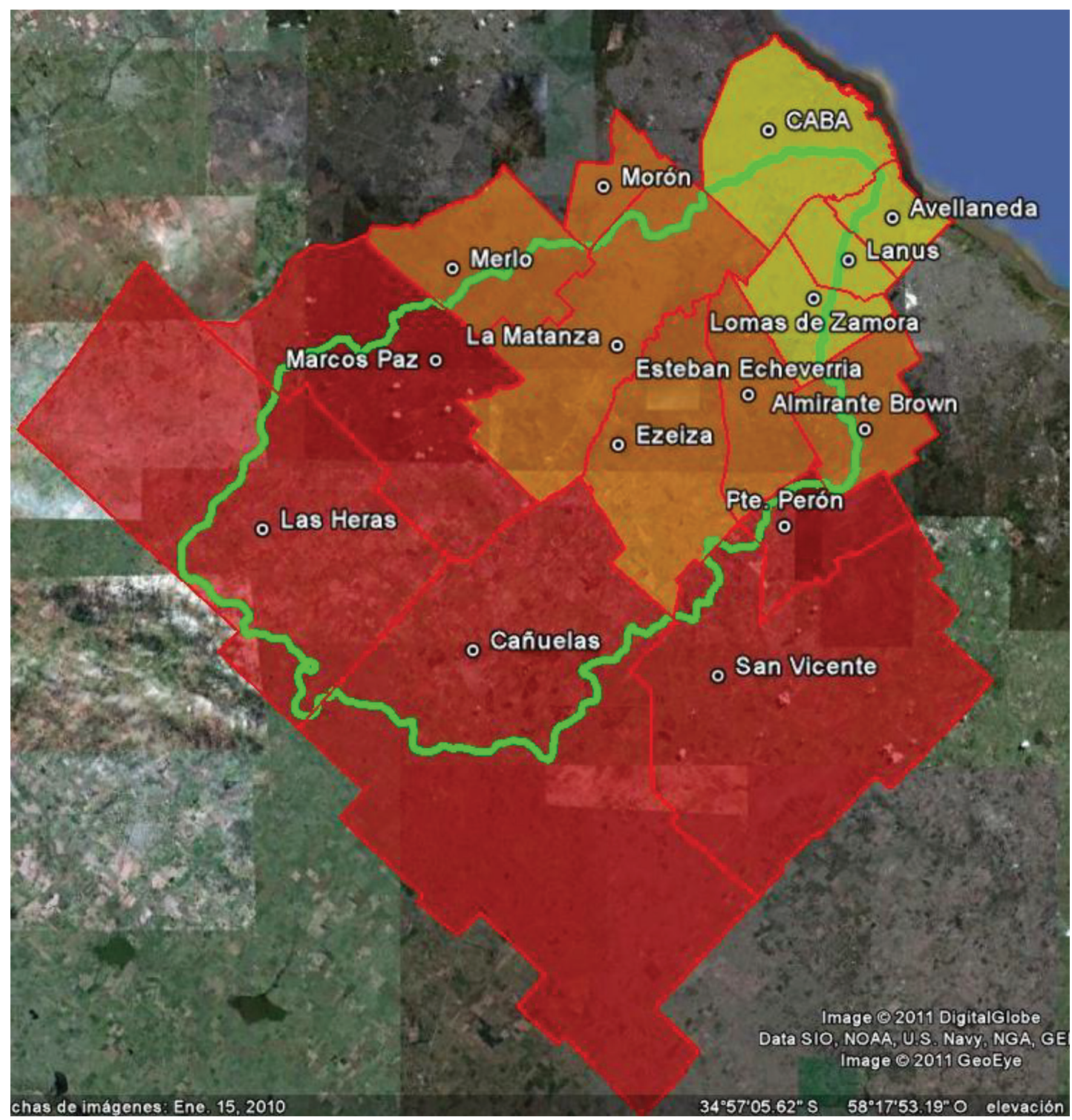

Fuente: Autoridad de la Cuenca Matanza Riachuelo (ACUMAR), 2011.

Según los datos del censo nacional del año 2010, el total de los habitantes en el área de la cuenca hidrográfica es de alrededor de 5 millones de personas, más del 10\% de la población argentina (Autoridad de Cuenca Matanza Riachuelo, ACUMAR, 2011). La CMR es la más contaminada del país y una 
de las más contaminadas del mundo. Según un informe elaborado por las organizaciones ambientalistas Green Cross International y el Blacksmith Institute, en 2013, la CMR se encuentra entre los diez lugares más contaminados del planeta (Blacksmith Institute, 2013, p. 10).

Pero uno de los aspectos más notables del informe de Green Cross International y el Blackmisth Institute, y abierto a la polémica, es que en un estudio previo realizado por dichas organizaciones en 2007, se ubicaba a la CMR dentro de los 30 lugares más contaminados del mundo (Blacksmith Institute, 2007). Dentro de este contexto surge la siguiente pregunta: ¿Es posible que los progresos en la institucionalización ambiental en la CMR no se vean reflejados en un avance objetivo en la salud del ecosistema de esta cuenca hídrica?

En el caso Mendoza, la Corte Suprema de Justicia Nacional (CSJN) generó un cambio institucional al implementar el paradigma ambiental a la gestión de la CMR. Sin embargo, a siete años del histórico fallo del 8 de julio de 2008, se pone en evidencia la fragilidad de la institucionalidad ambiental en la Cuenca.

Por ello, este trabajo tiene como objetivo analizar la fragmentación institucional y normativa como obstáculo para alcanzar mayor sostenibilidad, específicamente, en la gestión del agua. El análisis del caso de la CMR permite observar que la fragmentación jurídico-institucional trae como consecuencias el solapamiento normativo, la ineficacia del cumplimiento del derecho ambiental, obstaculiza la participación ciudadana y genera escenarios propicios a la corrupción.

\section{Métodos}

Este trabajo ha adoptado un diseño de tipo bibliográfico-documental, descriptivo y diacrónico.

La metodología utilizada es cualitativa, en particular, la hermenéutica jurídica. Se ha recurrido a diversas fuentes y técnicas de recolección de datos, y se consultaron estudios académicos, información estadística, fuentes judiciales, materiales oficiales y públicos. Como técnica de análisis se ha utilizado: la hermenéutica de los textos normativos y el análisis de documentos públicos.

\section{Marco Teórico}

\section{Contexto socio-histórico de la judicialización de la cuestión ambiental}

En el análisis del significado socio-político de la función judicial, Boaventura de Sousa Santos describe tres períodos fundamentales en las sociedades modernas: el período del Estado liberal, el del Estado de bienestar y el actual, que se podría designar como período de la crisis del Estado de bienestar.

El Período del Estado liberal comprende todo el s. XIX hasta la primera guerra mundial. En esta etapa el poder legislativo asume un claro predominio sobre los demás. El poder judicial es en la práctica neutralizado políticamente, a través del principio de legalidad, (prohibición de que los jueces decidan contra legem, y del principio de subsunción lógica de hechos a normas). Los jueces, por lo tanto, se desempeñan en un marco jurídico-político pre-constituido, por eso su poder es o funciona de manera retroactiva. Los jueces garantizan que el monopolio de la violencia sea ejercido con legitimidad. El poder judicial es reactivo, y sólo actúa cuando es instado por las partes o por otros sectores del Estado. Esta caracterización de los jueces en el período liberal revela su escaso peso político, como rama del poder público (De Sousa Santos, 2009, p. 80-81).

Con el desarrollo vertiginoso de la economía capitalista y el agravamiento consiguiente de las desigualdades emerge una creciente conflictividad social y, con ella, se definen las grandes divisiones políticas y sociales de la época. Sin embargo, "los jueces quedaron casi totalmente al margen de este proceso, dado que su ámbito de función se limitaba al microlitigio inter-individual, desligando de él el macrolitigio social” (De Sousa Santos, 2009, p. 81). Como conclusión, la institucionalidad del poder 
judicial de este período, predispuso a los jueces a una práctica judicial técnicamente exigente pero éticamente débil, inclinada a traducirse en rutinas (De Sousa Santos, 2009, p. 82).

En el período del Estado de Bienestar, la teoría de la separación de los poderes entra en crisis, sobre todo por la preeminencia del poder ejecutivo. Consiguientemente, el poder ejecutivo asume un rol mucho más activo en la producción del derecho. La sobrejuridicidad de la realidad social propia de este período pone fin a la coherencia y a la unidad del sistema jurídico. Surge un caos normativo que hace problemática la vigencia del principio de legalidad e imposible aplicar la subsunción lógica. El establecimiento de los derechos sociales y económicos lleva a juridificar a la justicia distributiva. El incremento de derechos es, en parte, consecuencia de la emergencia de actores colectivos como las organizaciones de trabajadores. A su vez, se dificulta el distingo entre litigios individuales y colectivos y se hace problemático, en la medida en que los intereses individuales aparecen de una u otra forma articulados con intereses colectivos.

La juridificación del bienestar social abrió el camino hacia nuevos campos de litigio en los dominios laborales, civiles, administrativos y de la seguridad social, lo que se tradujo en una explosión de litigiosidad. Asimismo, se abre un período de tensión en la neutralidad de los jueces. En este sentido cambia la orientación temporal del criterio para evaluar el desempeño de los jueces: pasa de ser retrospectivo a tener una importante dimensión prospectiva y centrada en sus efectos extrajudiciales con su consiguiente efecto de mayor visibilidad social y política de los jueces (De Sousa Santos, 2009, p. 83-84).

Finalmente, desde fines de la década de 1960 se abre un período de crisis del Estado de Bienestar, que perdura hasta la actualidad. El proceso de sobrejuridización de las prácticas sociales, que venía del período anterior, continuó acentuando la pérdida de coherencia y de unión del sistema jurídico.

Dos causas en particular complejas de este fenómeno son: la desregulación de la economía nacional en coexistencia con nuevos procesos de regulación a nivel internacional y trasnacional. Aparece aquí un nuevo factor asociado con el proceso de globalización de la economía en la fase informacional del capitalismo: la aparición de un nuevo derecho transnacional: la lex mercatoria en el derecho de los contratos internacionales, que coexiste con el derecho nacional aunque, a veces, se encuentran en contradicción con él (Zubizarreta Hernández, 2011, p. 195-196).

Sin embargo, la politización del poder judicial continúa en este período. Con la constitucionalización de los derechos de tercera generación, en especial los derechos ambientales y del consumidor surgen nuevas áreas de litigio. En estas áreas los jueces tienen poca preparación, no obstante, existen movimientos sociales con capacidad de movilizar a los magistrados, directa o indirectamente, a través de la incorporación a la agenda política o de creación de una opinión pública favorable. La politización de los tribunales reviste un carácter problemático debido a que la legitimidad del poder político de los jueces se fundamenta en el carácter apolítico de su ejercicio (De Sousa Santos, 2009, p. 91-92).

Otro aspecto fundamental de la politización del desempeño de los jueces se da respecto de su rol en el control social. Se trata de un aspecto, fundamentalmente, sensible ante el incremento de la corrupción política. Este fenómeno tiene entre sus causas principales el incremento de los contenidos sociales y económicos del Estado en el período del Estado de Bienestar. La regulación de la economía, la intervención del Estado en materia de obras públicas y la creación de la infraestructura requerida para efectivizar los derechos sociales y económicos llevó a expandir la administración pública y al incremento del presupuesto social y económico.

En este sentido, los vectores más decisivos de neutralidad o falta de neutralidad de los jueces son la voluntad política y la capacidad técnica en la lucha contra la corrupción. No resulta sorprendente que los jueces sean llamados al centro del debate político y se conviertan en un actor central de la crisis de la representación política, ya sea por contribuir a ella, eximiéndose de su responsabilidad de combatir el abuso del poder, o por contribuir a su solución, asumiendo esa responsabilidad (De Sousa Santos, 2009, p. 91-92). En caso de la judicialización del conflicto por el saneamiento y recomposición de la CMR, que se pasa a analizar a continuación, es un caso prototípico del contexto descripto en el nivel regional. 


\section{Contexto institucional-normativo}

La CMR ha sido considerada como el paradigma de la gestión fragmentada (Capaldo, 2011, p. 39). A lo largo de la Cuenca, se encuentran solapadas diversas competencias y jurisdicciones con distintos grados de autoridad sobre la misma. Las autoridades con jurisdicción sobre el área de la Cuenca son: el Gobierno Nacional; el Gobierno de la Ciudad Autónoma de Buenos Aires; el Gobierno de la provincia de Buenos Aires y 14 municipalidades: Almirante Brown, Avellaneda, Cañuelas, Esteban Echeverría, Ezeiza, General Las Heras, La Matanza, Lanús, Lomas de Zamora, Marcos Paz, Merlo, Morón, Presidente Perón y San Vicente.

Además, las autoridades con competencia sobre los cursos de agua de la Cuenca son: la Subsecretaría de Transporte por Agua y Puertos; el Puerto de Buenos Aires; la Prefectura Naval Argentina; la Dirección Provincial de Hidráulica y el Gobierno de la Ciudad Autónoma de Buenos Aires.

A su vez, tienen competencia sobre Cuenca, en calidad de sus efluentes líquidos, los siguientes organismos: el Instituto Nacional del Agua; el Entre Tripartito de Obras y Servicios Sanitarios; el Organismo Regulador de Aguas Bonaerenses; la Dirección Provincial Hidráulica; la Coordinación Ecológica Área Metropolitana Sociedad del Estado; la Administración General de Obras Sanitarias de la Provincia de Buenos Aires; el Ministerio de Salud y Acción Social de la Provincia de Buenos Aires; la Subsecretaría de Política Ambiental de la Provincia de Buenos Aires; el Gobierno de la Ciudad Autónoma de Buenos Aires; los gobiernos municipales de los 14 partidos con jurisdicción sobre la Cuenca y la Prefectura Naval Argentina.

En total son 39 organismos, los cuales deben aplicar unas 55 normas de diferente jerarquía que van desde la Constitución Nacional hasta las ordenanzas municipales. Ello sin incluir las diversas resoluciones, decretos y disposiciones específicas y/o temporales (Defensor del Pueblo de la Nación (DPN, 2003, pp. 33-34).

Respecto de la Nación, ésta tiene jurisdicción sobre la Cuenca por ser el Riachuelo un río navegable, en virtud del art. 75 inc. 10 de la Constitución Nacional. El art. 41 de la Constitución Nacional (CN) establece el derecho de todos los habitantes a un ambiente sano, equilibrado y apto para el desarrollo humano. El art. 43 legitima a los afectados, a ONGs inscriptas y defensores del pueblo para accionar vía de amparo en defensa del derecho constitucional al ambiente. El art. 124 establece el dominio originario de los recursos naturales existentes en su territorio.

A su vez, se aplican una serie de normas de presupuestos mínimos: Ley General del Ambiente 25675; Ley de Gestión Integral de Residuos Industriales 25612; Ley de Protección Ambiental para la Gestión Integral de los Residuos Domiciliarios 25916; Ley de Régimen de Gestión Ambiental de Aguas 25688; Ley de Régimen de Libre Acceso a la Información Pública Ambiental 25831. También son aplicables las leyes de Residuos Peligrosos 24051; Prevención de la Contaminación por buques 22190; Ley de navegación 20094; Contaminación atmosférica 20284; Condiciones de Seguridad e Higiene en el trabajo 19587; El Decreto 4516, Régimen de la Navegación Marítima, Fluvial y Lacustre.

El Código Civil (CC) tiene varios artículos aplicables a la CMR: (arts. 2513/4; 1113; 2499; 2616; 1132; 2618 y ss.; 2639/40; 2641; 2642; 2643/44, 2646; 2647 y ss.; 2645; 2540/3; 2547/8; 2164 y ss. у 4041(VALLS, 2012, p. 116-119) ${ }^{3}$. Mientras que el Código Procesal (CP) tipifica en su articulado algunos tipos penales relativos a casos de daño ambiental: arts. 79 y ss.; 89 y ss. 183; 184 incs. 2-4; 200; 203; 183; 184 incs. 2-4; 79 y ss.; 89 y ss.; 205-207; 182; 186-189; 189 bis; 202 y 203.

Se añaden los siguientes decretos: Decreto PEN 674/89 s/Protección de los Recursos Hídricos Superficiales y Subterráneos; Decreto PEN 776/92 (la Secretaría de Ambiente y Desarrollo Sustentable (SAyDS), que actualmente posee el rango de Ministerio, tiene el control de la contaminación de las aguas y la preservación de los recursos hídricos); Decreto PEN 999/92 sobre vertidos residuales o barros

3 Cabe mencionar que el Código Civil de la Nación ha sido modificado el $1^{\circ}$ de agosto de 2015, por lo que estos artículos que fueron aplicados para el caso de la CMR no se deberían haber aplicado en la actualidad. 
cloacales y la Resolución 634/98 SAyDS, sobre calidad ambiental para la franja costera del Río de la Plata y para los ríos Matanza y Riachuelo.

Con la finalidad de sancionar una norma que resuelva o minimice el problema de la gran cantidad de jurisdicciones y normas aplicables a la Cuenca, en 2006 se crea la Ley de la Cuenca MatanzaRiachuelo (ACUMAR) 26168.

Respecto a la Provincia de Buenos Aires, su jurisdicción sobre la CMR se encuentra incluida en la Carta Magna en el art. 121, y establece que las provincias conservan todo el poder no delegado al gobierno federal mediante la $\mathrm{CN}$ y el art. 124 dispone que corresponde a las provincias el dominio originario sobre sus recursos naturales. De modo concordante, el art. 28 de la Constitución de la Provincia de Buenos Aires, establece el dominio eminente sobre el ambiente y los recursos naturales en su territorio. Ese mismo artículo establece los derechos y deberes ambientales de los habitantes de la Provincia, y ordena controlar el impacto ambiental de todas las actividades que perjudiquen al ecosistema. El art. 36 reconoce el derecho a la salud, lo que implica que los ciudadanos bonaerenses tienen el derecho a consumir agua potable y a respirar el aire sin contaminantes. Finalmente, el art. 55 establece que el Defensor del Pueblo tiene a su cargo la defensa de los derechos individuales y colectivos de los habitantes.

Se consignan a continuación el resto de las normas del ordenamiento jurídico provincial aplicables a la CMR: Ley 11723; Ley 12257, Código de Aguas de la Provincia de Buenos Aires; Ley 13642/07- Adhesión Prov. de Buenos Aires a la ACUMAR; Ley 5965/58 y decretos reglamentarios s/ control de efluentes; Ley 13592/06 Gestión Integral de Residuos Sólidos Urbanos; Ley 11720/95 y decreto reglamentario de residuos especiales; Ley 11723, Protección, conservación, mejoramiento y restauración de los recursos naturales y del ambiente; Ley 11459 y decretos reglamentarios s/ Radicación industrial; Ley 12257/99, Código de aguas; Ley 10106 sobre drenajes rurales, desagües pluviales urbanos; Ley 9111 sobre disposición final de residuos sólidos urbanos; Ley 12814/94 Ampliación de la Reserva Natural Punta Lara; Ley 10907/90, de Reservas y Parques Naturales; Decreto-Ley 9867/82, de Conservación de Suelos; Decreto-ley 8912/77 s/ Ordenamiento territorial y uso del suelo; Decretos 2009/60 y 3395/96 sobre Protección a las fuentes de provisión y a los cuerpos receptores de agua y a la atmósfera; Res. 389/98 sobre calidad de vertidos; Res. 80/99 sobre establecimientos peligrosos de 3ra. Categoría; Res. 223/96 sobre control de industrias de 2da. Categoría.

Respecto a la Ciudad Autónoma de Buenos Aires, cabe tener en cuenta que su constitución establece su plena competencia y jurisdicción sobre la CMR en su art. 8. En el orden infraconstitucional las normas de la Ciudad de Buenos Aires aplicables a la CMR son: Ley 119/99 sobre manejo de los recursos naturales de la cuenca Matanza-Riachuelo; Ley 123/98 sobre evaluación de impacto ambiental (EIA); Ley 71/98- Creación del Consejo del Plan Urbano Ambiental (PUA); Ley 1227/03, de Patrimonio Cultural; Ley 1540/04, de control de la contaminación acústica; Ley 1356/04, de la Calidad Atmosférica; Ley 1854/06, Basura Cero; Ordenanza 39025, Código de prevención de la contaminación ambiental; Ordenanza 46956, de reforma del código anterior; Ordenanza 44485/90, Radicación Industrial; Decreto 1181/07, Código Ordenamiento Urbano; Res. 160/00, que crea la Unidad Ejecutora del Plan de la Cuenca Matanza-Riachuelo.

Por último, cabe considerar las instancias de coordinación inter-jurisdiccional en el ámbito territorial de la CMR: Convenio e/ Provincia de Buenos Aires y Municipalidad de la Ciudad de Buenos Aires p/ sanear el Riachuelo (Ley provincial 9597/80); Comité Ejecutivo del Plan de Gestión Ambiental y de Manejo de la Cuenca Hídrica Matanza-Riachuelo (Decreto PEN 1093/93); Decreto 1094/95, declara la liquidación del Comité Ejecutivo; Decreto PEN 482/95, crea al Comité Ejecutor del Plan de Gestión Ambiental y de Manejo de la Cuenca Hídrica Matanza-Riachuelo; Autoridad de Cuenca Matanza-Riachuelo (ACUMAR) creada por ley 26168/06. 


\section{El solapamiento normativo y de las competencias sobre la CMR en la gestión del agua}

La gestión de la CMR se encuentra signada por la fragmentación institucional, la coexistencia de 17 jurisdicciones (en los tres niveles de gobierno federal con aplicación de más de cincuenta normas) y la competencia de 39 organismos, en razón de su materia sobre el territorio de la cuenca. Esta situación ha generado una serie de dificultades y limitaciones en el ejercicio del poder de la policía, que corresponde a cada una de las jurisdicciones, debido a la carencia de instancias de coordinación que permitieran superar las dificultades institucionales.

En efecto, ese esquema de superposición de competencias, sumado a una profusa regulación normativa sujeta a modificaciones periódicas es producto de una serie de acuerdos institucionales fallidos. Esa compleja trama normativa ha contribuido a obstaculizar los controles en materia ambiental y sanitaria, creando un escenario propicio para la proliferación de actos corruptos. Algunos de los problemas de la fragmentación normativa-institucional que afectan al área de la CMR son transversales a la gestión del agua en el régimen metropolitano.

El caso de los Partidos de la Provincia de Buenos Aires que, actualmente, se encuentran incluidos en la concesión del servicio público del agua brindado por parte de la empresa Agua y Saneamientos Argentinos S.A. (AySA), resulta paradigmático debido a que es el único ámbito del país donde coexisten simultáneamente la jurisdicción nacional, provincial y municipal. Esto provoca que se superpongan normativas, y que dos o más organismos sean competentes para aplicar diferentes regulaciones y para controlar la calidad del servicio.

El Decreto Provincial 878/2003, capítulo V se refiere a las normas de calidad del agua. Dentro del mismo se creó una Comisión permanente de normas de potabilidad de vertido y efluentes líquidos y subproductos, que establece en cada región las características que debe tener el agua para que sea considerada potable y/o corriente. La clasificación existente para la Provincia de Buenos Aires sobre el agua corriente y el agua potable genera una problemática, puesto que esta clasificación no existe en el ámbito nacional ni de la Ciudad Autónoma de Buenos Aires.

La ley 26221 del año 2007, Anexo A y capítulo II punto 6 establece los parámetros exigidos para la calidad del agua para consumo humano, y en su capítulo II remite al marco regulatorio y a las normas aplicables. De acuerdo a la normativa anterior, puede afirmarse que existe un solapamiento legal entre la ley nacional 26221 de 2007 y el Decreto Provincial 878/2003, ya que ambos están vigentes para el ámbito de la Provincia de Buenos Aires.

La ley 26221 define en su art. 5 al agua potable como: "la que es apta para el consumo humano, la higiene personal, el uso doméstico habitual, adecuada a las normas mínimas de calidad establecidas en el presente Marco Regulatorio (Anexo A)". De esta forma, utiliza la técnica de la remisión legislativa y no aporta los parámetros de calidad aplicables al caso. Lo destacable es que el Decreto 878/2003 no fija ningún lineamiento de calidad, y únicamente crea una Comisión que se encarga de definir los parámetros de cumplimiento legal de la calidad exigible al servicio del agua. Esto acarrea un riesgo especial porque nunca se sabrá si los marcos legales establecidos por dicha Comisión son los adecuados para la protección de la salud de las personas, y si se aplicarán los parámetros internacionales o los nacionales.

En el art. 8 inc. j) del Decreto 878/2003 se mencionan las definiciones aplicables a la norma, y allí se establece que dicha Comisión será la autoridad que determinará las características que debe tener el agua para ser considerada potable. La categoría de agua corriente que está prevista para consumo humano, no cuenta con ningún parámetro legal de calidad exigible a las empresas que proveen la misma.

Además, este decreto ha creado dos categorías de agua que están permitidas para el consumo humano pero que poseen requisitos diferentes:

a) Agua Potable: Es la que cumple con todos y cada uno de los límites impuestos por la Comisión Permanente de Normas de Potabilidad y Calidad de Vertido de Efluentes Líquidos y Subproductos. 
b) Agua Corriente para el consumo humano e higiene: Es la que no cumple con algunos de los límites impuestos por la Comisión Permanente de Normas de Potabilidad y Calidad de Vertido de Efluentes Líquidos y Subproductos, pero su ingesta puede ser autorizada por períodos limitados.

En el inciso b) el término "algunos" aplicado a los límites de calidad del agua no ha sido definido y por lo tanto existe una laguna jurídica. Además, establece que el agua corriente puede ser consumida por "períodos limitados", que tampoco han sido determinados, y que pone en peligro la salud de los usuarios (cuya responsabilidad corresponde a la Administración Pública). Una de las consecuencias jurídicas que surgen de esta clasificación del agua para la Provincia de Buenos Aires, es que ambas pueden ser consumidas por seres humanos, a pesar de que cumplen distintos estándares de calidad. Esto implica que en la Provincia de Buenos Aires las exigencias para las empresas que proveen el servicio de agua son inferiores a las de otras jurisdicciones (como es el caso de la Ciudad de Buenos Aires). Esto también indica que los controles serán más leves, y lo mismo ocurrirá con el nivel de sanciones por incumplimiento u omisiones normativas.

La dificultad operativa surge en la práctica de la aplicación normativa porque estamos afirmando que en el mismo ámbito de concesión se deben aplicar normas diferentes. En la norma tampoco se explica qué parámetros de calidad no se deberían cumplir, evitándose así saber si esto es o no riesgoso para la salud humana. Por su parte, la Corte Suprema y otros tribunales, se han pronunciado sobre algunas de las obligaciones legales analizadas en este apartado (niveles de calidad del servicio de agua) durante los últimos años ${ }^{4}$.

\section{Judicialización del conflicto por el saneamiento y recomposición de la Cuenca Matanza- Riachuelo}

El caso Mendoza es el mayor precedente judicial de la historia nacional con respecto a causas colectivas ambientales (Lorenzetti, 2010, p. 49). Su origen es una demanda realizada por un grupo de 17 vecinos, (autos: "Mendoza, Beatriz Silvia y otros c/Estado Nacional y otros s/daños y perjuicios") que dicen padecer los efectos de la grave contaminación del área de la CMR, asimismo, demandan ante la Corte Suprema de Justicia de la Nación (CSJN) al Estado Nacional, a la Provincia de Buenos Aires, a la Ciudad Autónoma de Buenos Aires y a 44 empresas por daños y perjuicios y por la recomposición del daño ambiental. Se solicita por medio de esa acción un resarcimiento por el daño individual como por daño colectivo al ambiente (CSJN, M. 1569, L. XL. Sentencia. 20/06/2006).

Por otra parte, apoyándose en el art. 41 de la CN y en la ley general del ambiente (LGA) 25675, los vecinos reclamaron el cese de la contaminación y recomposición del daño ambiental colectivo de la Cuenca, con énfasis en la dimensión supraindividual del problema, como requisito fundamental para reparar el daño a la salud de habitantes (arts. 27, 28 y 30). Desde el punto de vista del proceso, la causa, que se inició como daños y perjuicios, en su transcurso no siguió un trámite ordinario sino, uno autónomo y urgente en el que se configuró un proceso ambiental atípico y expedito (Lorenzetti, 2010, p. 50).

El 20 de junio de 2006, la CSJN dicta una primera decisión sobre el caso Mendoza en la que decide:

a) no hacer lugar a la acumulación objetiva de los reclamos individuales de reparación referidos a los daños a las personas y a la propiedad. Reconoce, en cambio, su competencia para atender sobre la pretensión de prevenir y remediar el daño ambiental de la CMR;

4 Ver los siguientes casos jurisprudenciales: Boragina Juan Carlos, Miano Marcelo, Fabián y Ludica Juan Ignacio c/ Municipalidad de Junín sobre amparo" de 2009, "Spagnolo César Antonio contra Municipalidad de Mercedes sobre amparo" de 2008, y Florit Carlos Ariel y otros c/ Provincia de Buenos Aires y Aguas Bonaerenses S.A. sobre amparo" de 2010. 
b) ordena a los estados demandados a presentar un plan de remediación ambiental, que incluya las cuestiones de zonificación, control y evaluación ambiental sobre las industrias demandadas, educación ambiental e información pública;

c) ordena a las empresas demandadas a presentar información sobre la descarga de efluentes líquidos, plantas de tratamiento y seguro ambiental;

d) ordena a la parte actora a aportar a su escrito de demanda determinada información necesaria para dilucidar el caso;

e) no concede la medida cautelar solicitada por la parte actora;

f) convoca a una audiencia pública a todas las partes intervinientes fijada para el 5 de septiembre de 2006, con el objeto de que informen sobre los datos requeridos por el Alto Tribunal (CSJN. M. 1569. XL. Sentencia 20/06/2006).

En esa primera intervención y en las sucesivas etapas por las que atraviesa el caso Mendoza hasta la sentencia del 8 de julio de 2008, el Supremo Tribunal implementa una serie de innovaciones institucionales de carácter substancial en la historia de la institucionalización ambiental en Argentina. Un aspecto fundamental de ese proceso ha sido la utilización, en el transcurso del juicio, de las prerrogativas del art. 32 de la LGA:

a) emitir mandatos positivos a los otros poderes, lo que ha permitido a la Corte ordenar la adopción de un plan ante la omisión inconstitucional;

b) las facultades ordenatorias y la disección de pretensiones y de grupos demandados, lo que ha permitido a la Corte la formación de frentes para identificar y acumular las multipartes del expediente: el frente pasivo (integrado por las empresas demandadas) y el sector público (los estados demandados) y el frente activo (el DPN y los terceros intervinientes en la causa);

c) la Sentencia exhortativa, por la cual la Corte, con el fin de evitar la inconstitucionalidad por omisión en el futuro, les recuerda a los poderes responsables los contenidos que deberían estar presentes en los planes públicos (Nápoli \& Esain, 2008, p. 113).

El 8 de julio de 2008, a dos años de la declaración de competencia en el caso y sobre el material de prueba acumulado, el Alto Tribunal dicta la primera sentencia definitiva. Se destaca como una primera cuestión relevante en el fallo la reivindicación expresa de la Corte de su responsabilidad como intérprete final de la Constitución. Sobre el fundamento presentado en la demanda de 2004, la Corte reconoce el status constitucional del derecho al goce de un ambiente sano, establecido en el art. 41 de la CN, así como la obligación de recomponer el daño ambiental (CSJN, M. 1569, XL, 20/06/2006, Fallos: 326:2316, considerando 7). El derecho al ambiente como derecho humano, es cause y continente para los derechos individuales y sociales.

\section{La fragmentación jurídico-institucional como obstáculo a la participación ciudadana en la CMR}

En el área de la CMR se identifican obstáculos a la participación ciudadana asociados a las múltiples fragmentaciones de la gestión de la Cuenca. Una de ellas procede del daño a la salud derivado de la contaminación. En efecto, la fragmentación normativa, de las autoridades de control y de las políticas de agua incide en la falta o graves carencias en las actividades de control de los agentes contaminantes.

Esta situación es agravada por las condiciones sociales asociadas a la pobreza, que a su vez son transversales a vastas áreas de la Región Metropolitana de Buenos Aires (RMBA). Respecto a la gestión del agua potable, como vimos con anterioridad, el solapamiento normativo incide directamente en la falta de acceso al agua potable y saneamiento ambiental en vastos sectores urbanos. Se suma a ello, el hacinamiento, la escasez de espacios verdes, el acceso limitado a vacunas y medicamentos, las fallas 
en la recolección y disposición final de residuos sólidos domiciliarios e industriales, la localización inadecuada de algunas industrias, las fallas en el sistema de transporte público e inadecuado control de las emisiones del parque vehicular. Sin embargo, afirmaba el Defensor del Pueblo y Organizaciones Participantes en el Informe Especial sobre la CMR (2003) que:

...no existen estudios epidemiológicos realizados sobre la población de la cuenca, no hay datos confiables y comparables entre sí y las estadísticas de salud de los distintos partidos o municipios no se realizan con criterios comunes, y por lo tanto sus resultados son disímiles (DPN, 2003, p. 73).

Ese informe resalta, a su vez, que la carencia de estudios epidemiológicos obstaculiza el análisis de las relaciones de los datos ambientales con los datos de la salud. De esta forma, la población asentada en los márgenes del Riachuelo ha tenido obliterado el ejercicio del derecho a la salud, de los derechos sociales, del goce de un ambiente saludable y de la información pública ambiental. El estado de desinformación sobre la situación de la Cuenca contribuye a perpetuar una situación de inequidad en la que un sector de la población debe soportar los pasivos ambientales, mientras que los actores que los generan se favorecen de la carencia de controles y sanciones.

Respecto a los aspectos institucionales, la fragmentación de la gestión del agua y de la gestión metropolitana, se encuentra en relación con las características de las acciones ciudadanas en la Cuenca: se trata de acciones sumamente localizadas, esporádicas y con bajos niveles de incidencia.

La intervención de la CSJN en el asunto del Riachuelo marca un punto de inflexión respecto de la participación ciudadana en la Cuenca. El caso Mendoza no sólo es un caso bisagra en la política ambiental en Argentina, en términos institucionales - al exigir a los estados con jurisdicción en la Cuenca el saneamiento del ambiente - también lo es por sus aspectos socio-políticos: al establecer su competencia originaria sobre una cuestión que presentaba un proceso previo de participación de actores de la sociedad civil coordinados por el DPN.

La Corte Suprema acepta como terceros interesados en la causa al DPN (Res. 24/08/2006, según lo normado en los arts. $86 \mathrm{CN}$ y 30,31 y 32 Ley 25675) y a un grupo de ONGS de reconocida trayectoria en la problemática (Res. 30/08/2006, de conformidad con lo dispuesto en los arts. 90 del Código Procesal Civil y Comercial de la Nación (CPCC) y 30 ley 25675). En el proceso la Corte adopta un método de audiencias públicas otorgando, de esa forma, gran relevancia a la participación ciudadana en un proceso abierto y participativo. Más adelante, en el fallo del 8 de julio de 2008 formaliza esa participación de los terceros interesados al encargar al Defensor del Pueblo que:

\footnotetext{
...conformará un cuerpo colegiado con los representantes de las organizaciones no gubernamentales que intervienen en la causa en igual carácter de terceros, coordinando su funcionamiento y distribuyendo internamente las misiones, entre las que se incluyen la recepción de información actualizada y la formulación de planteos concretos ante la Autoridad de Cuenca para el mejor logro del propósito encomendado según criterios de igualdad, especialidad, razonabilidad y eficacia (CSJN, M.1569.XL- 08/07/2008)
}

En materia de información pública, la CSJN ha entendido, que su acceso irrestricto es un aspecto de enorme importancia. En ese sentido y en el ámbito de la causa judicial Matanza-Riachuelo, exige conformar en un plazo de 30 días hábiles, un "sistema de información pública a través de Internet, para el público en general, de modo concentrado, claro y accesible" (CSJN, M.1569.XL- 08/07/2008, considerando 17, punto II), cuyo incumplimiento "importará la aplicación de una multa a cargo del presidente de la Autoridad de Cuenca” (CSJN, M.1569.XL- 08/07/2008, considerando 17, punto II).

Ese proceso no estaba exento de obstáculos, existen algunos problemas conexos como la carencia de mecanismos de participación institucionalizada. Uno de los aspectos más polémicos de la gestión de la ACUMAR ha sido su estrategia de control de las fuentes de contaminación, lo cual se refleja en las Resoluciones ACUMAR 1/2007, 2/2007,3/2009, 278/2010 y 366/2010. 
La Resolución ACUMAR 3/2009, relativa a los usos y objetivos de calidad del agua, que establece el uso IV, como meta a alcanzar de mediano a largo plazo - apta para actividades recreativas pasivas -, ha sido objeto de varias críticas: a) por no promover la rehabilitación de las condiciones que constituyen el ecosistema; b) por carecer de progresividad (art. 4 de la Ley 25675); c) por homogeneizar una cuenca diversa; d) por tolerar el vertido de sustancias peligrosas y e) por no haber sido objeto de participación social (arts. 19 y 20 de la ley 25675 (DPN, 2015, p. 14-15). Justamente, el funcionamiento de la Comisión de Participación Social es otro de los aspectos que ha sido objeto de críticas.

Según Malpartida, el uso IV, desde un punto de vista ecológico, es inapropiada su aplicación de forma uniforme a toda la Cuenca, dado que resulta muy exigente para algunos puntos, como la Cuenca baja pero "condena a malograr definitivamente la cuenca media y alta" (Malpartida, 2012, p. 94). Cabe tener en cuenta que, según los muestreos - cuya sistematización es reciente - la calidad de las aguas de la Cuenca es muy variable. Que esos resultados son esperables debido a la heterogeneidad de la Cuenca: abarca una superficie de $2200 \mathrm{~km}^{2}$, con 17 ríos y arroyos distintos (Martínez, 2012, 103). La homogeneización de la norma habilitaría, en consecuencia, que se degraden zonas que actualmente presentan condiciones ambientales aceptables (DPN, 2013, p. 11).

$\mathrm{Al}$ establecer una meta de calidad del agua ambientalmente permisible, de manera implícita se condicionan muchas de las dimensiones cruciales de la gestión ambiental: el control de la contaminación industrial, el tratamiento de efluentes cloacales, la gestión de los residuos sólidos, el ordenamiento ambiental del territorio, el control de actividades agropecuarias, la protección de márgenes (DPN, 2013, p. 10).

Se añade que, esa norma no fue objeto de participación social. Teniendo en cuenta que se trata de una medida de política ambiental a largo plazo, deben incluirse los mecanismos de participación ciudadana previstos en los arts. 19 y 20 de la Ley 25675 (DPN, 2015, p. 16). Ante ese diagnóstico propone el DPN y el Cuerpo Colegiado:

\footnotetext{
Modificar la Resolución ACUMAR N 3/2009 de modo que establezca una progresividad en los objetivos a alcanzar y responda a las distintas realidades existentes al interior de la cuenca. La inclusión de la totalidad de las sustancias relevantes y la participación social en su elaboración son requisitos ineludibles para ello (DPN, 2013, p. 7)
}

\section{Reflexiones finales}

La problemática socio-ambiental de la Cuenca Matanza-Riachuelo es la más grave del país y es una de las más críticas del mundo.

En el ámbito argentino, el escenario institucional es sumamente fragmentado, con casi inexistentes niveles de coordinación entre las diversas jurisdicciones y los organismos con competencia en la Cuenca. Esta situación ha dificultado, durante varias décadas, la determinación de las responsabilidades, y ha dado lugar a una serie de disfuncionalidades institucionales.

El análisis de la situación normativa-institucional en la CMR, en relación con sus jurisdicciones y competencias sobre el aire, el agua y el suelo permite reconocer la existencia de una profusa normativa: aproximadamente 55 normas en diferentes niveles y jerarquías. El caos normativo resultante de ese contexto ha dado lugar a una maraña de arreglos institucionales fallidos generando situaciones de corrupción en varias dimensiones: carencia u obstáculos a los controles de efluentes contaminantes; carencias en la realización de estudios epidemiológicos en la zona; dificultades en el acceso a la información y en el derecho a peticionar y tener respuestas en tiempo y forma por parte de las autoridades; el derecho a la participación ciudadana, entre otros.

En este trabajo se han analizado dos consecuencias cruciales de la fragmentación jurídico-institucional: los obstáculos a la participación ciudadana y el solapamiento normativo.

Respecto a la participación ciudadana, el repaso de la normativa permite reconocer la existencia de un amplio marco legislativo de derecho a la participación ciudadana en materia ambiental. Sin 
embargo, no se ha logrado garantizar el ejercicio efectivo del derecho a participar debido a su deficiente institucionalización, especialmente, en los niveles de decisión y control, por la falta de una gestión eficaz por parte de las autoridades, y por graves carencias en aspectos que constituyen condiciones necesarias para la participación, como brindar información pública en términos veraces ante reiterados pedidos (DPN, 2005, p. 89-90). Otro de los factores que obstaculizan el ejercicio de las acciones ciudadanas es la clara ausencia de una política pública ambiental.

Los dos ámbitos en los cuales se ha detectado un solapamiento con respecto a la regulación de la calidad del servicio del agua, para los ámbitos de la Ciudad Autónoma y de la Provincia de Buenos Aires son los siguientes:

- Definición del agua potable.

- Parámetros de calidad del agua.

Los mismos atentan contra el principio de seguridad jurídica, creando incertidumbre en los usuarios del servicio e imprecisiones en el accionar de los organismos de control.

El contexto de superposición de competencias y solapamiento normativo se encuentra estrechamente asociada a la falta de eficacia del derecho ambiental, que también es otro factor que afecta al derecho a la participación ciudadana. Una investigación cualitativa basada en entrevistas a testigos clave (jueces, agentes de la administración pública y legisladores), muestra una consideración baja del acceso a la justicia y a la información ambiental (Capaldo, 2011, p. 29-30).

La Corte Suprema, al intervenir en la causa Mendoza el 20 de junio de 2006, abre un proceso deliberativo al convocar audiencias públicas para que comparezcan los demandados (Estados con jurisdicción en la Cuenca y 44 empresas), pero también ordena al Estado Nacional, a la Provincia de Buenos Aires, a la Ciudad de Buenos Aires y a la Coordinación Federal Ambiental (COFEMA) para que, en los términos de la Ley General del Ambiente 25675 presentasen un plan de recomposición.

Los estados demandados, representados por la SAyDS presentaron un Plan Integral de Saneamiento Ambiental y para ejecutarlo crearon la ACUMAR, con la finalidad de resolver o minimizar el problema de la multiplicidad de jurisdicciones y normas que actúan sobre la CMR. Sin embargo, a siete años del histórico fallo se evidencian las dificultades para desestabilizar los obstáculos que impiden la coordinación inter-jurisdiccional para impulsar procesos de cambio de larga duración. A fines de 2013, el juez a cargo de la ejecución de la sentencia, Luis Armella, fue apartado de su cargo y el proceso se ralentizó. Se designó al Juzgado Nacional en lo Criminal y Correccional Federal $\mathrm{N}^{\circ} 12$ a cargo del Dr. Sergio Torres, para la inspección de los contratos y de su nivel de ejecución presupuestaria, y se delegaron todas las restantes competencias atribuidas en la sentencia del 8 julio del 2008 en el Juzgado Federal en lo Criminal y Correccional № 2 de Morón, cuyo titular es el Dr. Jorge Rodríguez (CSJN, M.1569.XL,19/12/2012, Considerando 4). La nueva distribución de responsabilidades complejiza la búsqueda de información sobre el proceso judicial, más allá de las actas y resoluciones de los respectivos jueces.

A su vez, la acefalía de la Defensoría del Pueblo, desde diciembre del 2013, pone en peligro la continuidad del Cuerpo Colegiado, debido a que el Defensor del Pueblo es el único legitimado activo para actuar en la causa y coordinador del Cuerpo Colegiado.

Además de los aspectos jurídicos e institucionales, los socio-culturales también son de gran importancia, dada la magnitud de la problemática, también se deben tener en consideración a las percepciones del riesgo y a los patrones de comportamiento insustentables fuertemente arraigados. Esas percepciones debilitan la progresión hacia la institucionalización de comportamientos pro-ecológicos, a su institucionalización jurídica y política, y a la eficacia de la política y del derecho ambiental. 


\section{Bibliografía}

ACUMAR. Plan Integral de Saneamiento Ambiental de la Cuenca Matanza Riachuelo. Buenos Aires, 2011, disponible en: www.acumar.gov.ar/Informes/Control/CalAmb/Abril2011/Abril2011_link. pdf (acceso el 12 oct. 2014).

Constitución de la Ciudad Autónoma de Buenos Aires, BO: 1 ene. 1996.

Constitución de la Provincia de Buenos Aires, BO: 13 sept. 1994.

Blacksmith Institute. Annual Report 2013.

The World's Worst Polluted Places. The Top Ten of the Dirty Thirty. The Remainder of the Dirty Thirty. 2007. Disponible en: http://www.blacksmithinstitute.org/articles/file/47.pdf (consultado el 4 mar. 2013).

Capaldo, Griselda. 2011. Gobernabilidad Ambiental y Eficacia del Derecho: dos magnitudes del desarrollo sustentable. En (Griselda Capaldo (comp.), Gobernanza y Manejo Sustentable del Agua - Governance and sustainable Management of Water, pp. 165-191. Buenos Aires: Mnemosyse.

Convención Interamericana Contra La Corrupción (CICC).

CSJN. Sentencia “Mendoza, Beatriz Silvia y otros c/Estado Nacional y otros s/daños y perjuicios (daños derivados de la contaminación ambiental del Río Matanza-Riachuelo)" (M.1569.XL)- fecha 20 de Junio de 2006.

CSJN. Sentencia "Mendoza, Beatriz Silvia y otros c/Estado Nacional y otros s/daños y perjuicios (daños derivados de la contaminación ambiental del Río Matanza-Riachuelo)" (M.1569.XL)- fecha 08 de Julio de 2008.

CSJN. Sentencia "Mendoza, Beatriz Silvia y otros c/Estado Nacional y otros s/daños y perjuicios (daños derivados de la contaminación ambiental del Río Matanza-Riachuelo)" (M.1569.XL), Resolución del 19 de diciembre de 2012.

Decreto 878, Aprueba el marco regulatorio para la prestación de los servicios públicos de provisión de agua potable y desagües cloacales en la Provincia de Buenos Aires, 2003.

De Sousa Santos, Boaventura. 2009. Sociología Jurídica Crítica. Para un nuevo sentido común en el Derecho. Bogotá: Instituto Latinoamericano de Servicios Legales Alternativos.

DPN. Informe especial sobre la Cuenca Matanza-Riachuelo 2003.

DPN. Informe Especial de Seguimiento Cuenca Matanza - Riachuelo (2003/2005).

DPN. Informe Especial del Cuerpo Colegiado (enero 2013). Caso Matanza Riachuelo: bases para un adecuado cumplimiento del fallo de la Corte Suprema de Justicia de la Nación. Buenos Aires, 2013.

Herrero, Ana, \& Fernández, Leonardo. 2008. De los ríos no me río. Cuencas Metropolitanas de Buenos Aires. Buenos Aires: Temas.

Informe Especial del Cuerpo Colegiado, julio de 2015.

Ley 25675, Ley General del Ambiente, BO: 28/11/2002.

Ley de Creación de la ACUMAR 26168, BO: 05/12/2006.

Lorenzetti, Ricardo. 2010. Justicia Colectiva. Buenos Aires: Rubinzal - Culzoni.

Malpartida, Alejandro. 2002. La Cuenca Matanza Riachuelo. Revisión de Antecedentes de Recursos Naturales. Compuestos xenobióticos y otros polutantes en la cuenca. Buenos Aires: UTN- Multimedios Ambiente Ecológico.

Malpartida, Alejandro. 2012. La Cuenca Matanza-Riachuelo a la luz de las acciones de la ACUMAR 2006-2012. La Corte Ordenó Recomponer el Riachuelo. Buenos Aires: Asociación de Vecinos de la Boca.

Martínez, Enrique. 2012. Evaluación de las condiciones ambientales fijadas a la industria por la ACUMAR. La Corte Ordenó Recomponer el Riachuelo. Buenos Aires: Asociación de Vecinos de la Boca. 
Nápoli, Andrés \& Esain, José. 2008. Riachuelo. Habemus Sentencia. Revista de Derecho Ambiental, volumen 16, Buenos Aires: AbeledoPerrot.

ONU. Convención de las Naciones Unidas contra la Corrupción (CNUCC).

Valls, Claudia. 2012. Normas aplicables a la Cuenca Matanza-Riachuelo. Contaminación fluvial. Temas sudamericanos. Del Castillo, Lilian (comp.), Contaminación Fluvial. Temas sudamericanos. Buenos Aires: La Ley.

Zubizarreta Hernández, Juan. 2011. Un nuevo Derecho Corporativo Transnacional. El carácter feudal de los ordenamientos jurídicos globales. En: Capaldo, Griselda (ed.), Gobernanza y Manejo Sustentable del Agua - Governance and sustainable Management of Water, pp. 195-215.Buenos Aires: Mnemosyse. 
